\title{
Statement of the ESHG on direct-to-consumer genetic testing for health-related purposes
}

\author{
European Society of Human Genetics*
}

\begin{abstract}
Many private companies offer direct-to-consumer (DTC) genetic testing services. Some tests may detect severe and highly penetrant monogenic disorders, while other tests are for genetic variants found associated with increased susceptibility for common and complex diseases in large-scale population studies. Through its Public and Professional Policy committee followed by member and expert consultation, the European Society of Human Genetics has developed the following policy on advertising and provision of predictive genetic tests by such DTC companies: (1) clinical utility of a genetic test shall be an essential criterion for deciding to offer this test to a person or a group of persons; (2) laboratories providing genetic tests should comply with accepted quality standards, including those regarding laboratory personnel qualifications; (3) information about the purpose and appropriateness of testing should be given before the test is done; (4) genetic counselling appropriate to the type of test and disease should be offered; and for some tests psychosocial evaluation and follow-up should be available; (5) privacy and confidentiality of sensitive genetic information should be secured and the data safely guarded; (6) special measures should be taken to avoid inappropriate testing of minors and other legally incapacitated persons; (7) all claims regarding genetic tests should be transparent; advertisement should be unbiased and marketing of genetic tests should be fair; (8) in biomedical research, health care and marketing, respect should be given to relevant ethical principles, as well as international treaties and recommendations regarding genetic testing; and (9) nationally approved guidelines considering all the above-mentioned aspects should be made and followed.
\end{abstract}

European Journal of Human Genetics (2010) 18, 1271-1273; doi:10.1038/ejhg.2010.129; published online 25 August 2010

Progress in biotechnology and genetic research has led to an increasing number of tests with potential predictive health information. In parallel with this development, private companies have established direct-to-consumer (DTC) genetic testing services, both for monogenic and severe genetic disorders and for genetic variants possibly associated with common complex diseases (susceptibility variants). Tests are also offered for conditions of minor or no health importance.

The European Society of Human Genetics (ESHG) is concerned about the way in which commercial companies are currently introducing genetic tests into the market outside of the scope of the traditional healthcare system. With this Statement, we provide a formal policy with regard to DTC advertising and provision of genetic tests with predictive health information. Important issues, such as DTC paternity and ancestry testing, are thus outside the scope of this Statement.

In line with the Council of Europe's Additional Protocol to the Convention on Human Rights and Biomedicine, concerning Genetic Testing for Health Purposes and the OECD Guidelines for Quality Assurance in Molecular Genetic Testing, this Statement highlights the importance of right to information, quality of the test performed, clinical usefulness of the tests provided, the need for individualized medical supervision, the provision of pre-test information and genetic counselling, follow-up and support in the interpretation of results and their psychosocial impact, the protection of persons not able to consent, respect for privacy and confidentiality, and the storing of the samples, their property and respect for ethical principles in research.

\section{RIGHT TO GENETIC INFORMATION}

Individuals are entitled to health information and genetic information about themselves. However, this right to know must be exercised with due respect for the need to protect the same individuals from inappropriate genetic information and testing. Various companies hold out that DTC genetic testing enhances an individual's autonomy and control over his or her future. ESHG underlines that this can occur only when consumers are offered adequate pre-test information, including counselling and psychosocial support when appropriate, and when the tests offered are of good quality and medically relevant. Otherwise, valid interpretations and specific recommendations to promote the health of the individual cannot be made.

\section{DTC ADVERTISING OF GENETIC TESTING}

Research on DTC advertising of prescription medicine has shown that this has created an inappropriate demand for medications. Moreover, it has shown that various advertisements for drugs have been misleading. Overstatement of effectiveness or minimization of risk has led to inadequate or inappropriate changes in medication, diet or lifestyle by consumers. DTC advertising of genetic tests for healthrelated purposes runs the same risks as DTC advertising of prescription medicine in this regard. Aggressive marketing strategies and slogans for DTC genetic testing might overstate the potential for predictive information of such tests and overrate its future health implications. All this is likely done to increase test uptake and artificially create new demands, that is, to earn money. We strongly believe that every advertisement should conform to the 
same international standards and guidelines that apply for advertisement of drugs and medical devices. Among other issues, the advertisement should be accurate and not misleading, claims should be transparent and supported by current evidence, and complete and accurate information about the test limitations, risks and benefits should be provided.

\section{QUALITY OF GENETIC TESTING SERVICES}

The ESHG stresses the importance of ensuring high quality of genetic testing services. This includes (1) the quality of the genetic tests (eg, their analytical validity and clinical validity and utility); (2) the quality of the genetic testing laboratories (as laid out in OECD recommendations, see http://www.oecd.org); and (3) proper qualifications, training and continuous education of the personnel involved in the provision of the services.

Many of the tests currently offered by DTC have been based on an association between a specific genetic variant and a particular disorder. It is of particular concern that their predictive value must be sufficient to meet the standards for clinical use. The clinical utility of a genetic test should be an essential criterion for deciding to offer this test to a person or a group of persons. ESHG endorses generally accepted criteria for analytical validity, and clinical validity and utility of genetic tests, as those being developed by the UK Genetic Testing Network (Gene Dossiers) or the EU-funded Eurogentest network of excellence (Gene Cards). Furthermore, the ethical, legal and social implications of the tests provided should be considered extensively in all of its phases (research, development and transfer into clinical practice).

In light of these criteria, the ESHG opposes the premature DTC commercialization of various genetic tests. For many recently developed tests, evidence for clinical utility is not available. For tests on genetic variants with potential predictive value, controlled unbiased studies must be done to generate such evidence for clinical utility, and the result may even be population specific. Tests where clinical utility is unproven but seems likely should be performed in the context of the healthcare system and be subject to adequate post-marketing surveillance.

All laboratories offering genetic testing services should implement an internal quality system and be subject to regular external quality assessment. Certification of laboratory procedures and accreditation of the tests offered is the standard for guaranteeing the quality of molecular genetic testing for health purposes. All persons involved in the provision of genetic services (ie, medical doctors, nurses, genetic counselors, and biologists and technicians working at the laboratories) should have the appropriate qualifications and training and should perform their role in accordance with professional best practices and ethical standards.

\section{INDIVIDUALIZED MEDICAL SUPERVISION}

The offer of genetic tests providing health-related information, in the absence of clinical indications and individualized medical supervision, may compromise patient health. Key concerns are the provision of sufficient information about the purpose and appropriateness of testing, its possibilities and limitations, as well as the clinical significance of testing. An involvement of independent medical professionals could avoid the waste of money on tests that are clinically irrelevant. In addition, the cost and adverse psychosocial effects of unnecessary follow-up or medical investigations could be avoided.

As more and more medical professionals are confronted with the results of genetic testing, continued education to understand the usefulness of such tests is increasingly important. Such training should also be offered to general practitioners, public health physicians and students. In parallel, improved genetic education of the public in general and the media in particular will be of great importance to foster medical supervision of health-related genetic testing.

\section{PRE-TEST INFORMATION AND GENETIC COUNSELLING}

The labelling information on genetic tests must be true, accurate, accessible, complete and comprehensible. Although some companies have websites providing most or all of this, the information is often of promotional nature and intended to sell tests, which might compromise the truth in labelling.

Genetic counselling is the process through which information enables individuals to make their own free decisions about testing. A website cannot replace appropriate pre-test and post-test genetic counselling, which usually involves a face-to-face consultation with a knowledgeable professional. Genetic counselling is a communication process, which deals with the occurrence, or risk of occurrence, of a genetic disorder in the family. The process involves an attempt by appropriately trained person(s) to help the individual or the family to understand the medical facts of the disorder and the options on how to deal with it. A conflict of interest may arise when the healthcare professionals involved in the counselling are employed by or are linked to the companies selling the tests. In this case impartial health advice might be compromised.

\section{INFORMED CONSENT}

A predictive genetic test for health purposes may only be carried out after obtaining the individual's free and informed consent. Although DTC genetic testing companies usually require a consent form to be signed when ordering a test, the ESHG considers that an informed consent procedure cannot be reduced to sign a consent form. Although such text might be necessary to document the process of informed consent, it cannot be a substitute for the process that needs to be in place to ensure that individuals understand the disclosed information, are legally competent and cognitively capable of acting without external pressure, and give their agreement to all the elements involved. Privacy and confidentiality of the results, as well as possible consequences related to their disclosure to third parties, such as insurance companies and employers, should be discussed, when appropriate, as well as the property of the biological material and its fate after the results are confirmed.

Moreover, any genetic testing service that requires a sample to be collected at home runs the risk of samples being submitted for testing without obtaining proper consent or without even the knowledge of the person to whom it pertains. DTC genetic testing companies do not have the necessary mechanisms in place to ensure that the biological sample provided for testing is obtained from the person claimed to be the sample provider.

\section{GENETIC TESTING IN MINORS}

The principles set out in the recommendations for genetic testing on minors, already issued by the ESHG, apply here as well. The very context of DTC genetic testing does not allow for an adequate assessment of the competence of a minor. Therefore, the ESHG considers that DTC genetic tests should not be offered to individuals who have not reached the age of legal majority.

\section{RESPECT FOR PRIVATE LIFE}

Any genetic test should always be performed with respect for private life. In particular, companies offering DTC genetic tests should 
preserve the customer's privacy, keep their data confidential, inform them about their security procedures, explain what will happen to the sample and the data when the testing process is concluded, and have a clearly laid-out plan as to what will happen to the samples and data should the company be sold or go bankrupt. Companies inviting their customers to share their genetic information via a web community or forum should inform people about the potential risks for disclosure of this type of sensitive information. The ESHG disapproves any use of personal details or genetic information by test providers (or other companies) for DTC marketing of medicines, vitamins or dietary supplements.

\section{RESEARCH}

Various companies offering DTC genetic services perform research activities on the biological samples and information of their customers. The ESHG is concerned with the inadequate consent process through which customers are enrolled in such research. If samples or data are to be used in any research, this should be clear to consumers, and a separate and unambiguous consent procedure should take place. Informed consent documents for participation in research should disclose the procedures for storing and disposal of samples and genetic information, the time period and conditions for storing them, inform participants of the identity of any third parties who may be granted access to data or samples, and include also information on the fact that the research may lead to commercialization and patents, on any customers' rights to commercial benefits and on the property of biological samples and data.

\section{OVERSIGHT OF GENETIC TESTING}

In order to prevent premature translation of genomic services into the market or clinical practice, a regulatory oversight will be required. Oversight will be important to synthesize available evidence on the clinical validity and utility of emerging genetic tests and to identify current gaps in knowledge, as well as the studies and measures needed to resolve them.

National legislation that requires a responsible medical person to be involved before a genetic test is provided has been realized in some European countries and should be encouraged in others. At the European level, regulatory mechanisms, and in particular the adaptation of the European In Vitro Diagnostic Devices Directive (to consider genetic tests of being high risk), the implementation of the Additional Protocol to the Convention on Human Rights and Biomedicine, concerning Genetic Testing for Health Purposes, and the implementation of the OECD Council Recommendation on Quality Assurance in Molecular Genetic Testing will be necessary to ensure appropriate introduction of genetic tests into the healthcare system.

\section{IMPACT ON THE HEALTHCARE SYSTEM}

The provision of genetic testing services for health-related purposes outside the healthcare system may lead to an increased burden on public health resources. Increased calls on primary-care physicians or clinical genetic services are already one consequence. An undesirable impact on patient health, sometimes due to misinterpretation of complex genetic information, may have even greater healthcare consequences. The ESHG advocates the introduction of genetic tests into the healthcare system only if these tests meet the necessary criteria established by the Member States and European Union health authorities and regulatory agencies, and the necessary quality criteria. The ESHG urges that only genetic tests with proven or obvious clinical utility should be reimbursed by social security systems. Equitable access to such tests is also important.

\section{CONCLUSION}

DTC genetic testing and the advertisement of genetic tests of unproven benefit or without adequate independent genetic counselling are in opposition to the professional standards the ESHG sustains. Furthermore, such DTC testing might negatively influence the perception of genetic testing and the tests' usefulness for health care. Legislators, regulators and policy makers are urged to protect the population by adapting the European IVD Directive and implementing the OECD Council Recommendations on Quality Assurance in Molecular Genetic testing, and the Council of Europe Additional Protocol to the Convention on Human Rights and Biomedicine, concerning Genetic Testing for Health Purposes.

Members of the Public and Professional Policy Committee in the first half of 2010 were: Pascal Borry (corresponding author), Martina C Cornel (chair), Nurten Akarsu, Anne Cambon-Thomsen, Francesca Forzano, Shirley Hodgson, Ulf Kristoffersson, Jan Lubinski, Christine Patch, Jorge Sequeiros, Aad Tibben and Lisbeth Tranebjaerg. The PPPC was supported by Carla van El. A draft document developed by the PPPC was circulated to the ESHG board and membership in April 2010. An adapted version was circulated to selected experts in May 2010. The Board approved the recommendations on 12 June 2010.

\section{REFERENCES}

1 Additional Protocol to the Convention on Human Rights and Biomedicine, concerning Genetic Testing for Health Purposes. Council of Europe, Strasbourg, 2008. Available from http://conventions.coe.int/Treaty/EN/Treaties/Html/203.htm.

2 Ashley EA, Butte AJ, Wheeler MT et al: Clinical assessment incorporating a personal genome. Lancet 2010; 375: 1525-1535.

3 Brand A: Integrative genomics, personal-genome tests and personalized healthcare: the future is being built today. Eur J Hum Genet 2009; 17: 977-978.

4 Directive 98/79/EC of the European Parliament and of the Council of 27 October 1998 on in vitro diagnostic medical devices. Available from http://eur-lex.europa.eu/ LexUriServ/LexUriServ.do?uri=CELEX:31998L0079:en:HTML.

5 European Society of Human Genetics: Genetic testing in asymptomatic minors: recommendations of the European Society of Human Genetics. Eur J Hum Genet 2009; 17: 720-721.

6 Human Genetics Commission: A common framework of principles for direct-toconsumer genetic testing services. Consultation document, 2010. Available from http:// www.hgc.gov.uk/UploadDocs/Contents/Documents/Principles\%20consultation\%20final.pdf.

7 Kuehn BM: NIH launching genetic test registry. JAMA 2010; 303: 1685.

8 Lwoff L: Council of Europe adopts protocol on genetic testing for health purposes. Eur J Hum Genet 2009; 17: 1374-1377.

9 OECD Guidelines for Quality Assurance in Genetic Testing: Organisation for Economic Co-operation and Development, 2007. Available from http://www.oecd.org/dataoecd/ 43/6/38839788.pdf.

10 Patch C, Sequeiros J, Cornel MC: Genetic horoscopes: is it all in the genes? Points for regulatory control of direct-to-consumer genetic testing. Eur J Hum Genet 2009; 17: 857-859.

11 Patch C, Sequeiros J, Cornel MC: Direct to consumer genetic tests. Eur J Hum Genet 2009; 17: 1111.

12 Van Ommen GB, Cornel MC: Recreational genomics? Dreams and fears on genetic susceptibility screening. Eur J Hum Genet 2008; 16: 403-404. 